\title{
A preliminary study on an image analysis based method for lowest detectable signal measurements in Pulsed Wave Doppler ultrasounds
}

\author{
Giorgia Fiori $^{1}$, Fabio Fuiano ${ }^{1}$, Andrea Scorza $^{1}$, Jan Galo ${ }^{2}$, Silvia Conforto ${ }^{1}$, Salvatore A. Sciuto ${ }^{1}$ \\ ${ }^{1}$ Engineering Department, ROMA TRE University, Via della Vasca Navale 79, 00146 Rome, Italy \\ ${ }^{2}$ Clinical Engineering Service, IRCCS Children Hospital Bambino Gesù, Piazza di Sant'Onofrio 4, 00165 Rome, Italy
}

\begin{abstract}
Nowadays, Doppler system performance evaluation is a widespread issue because a shared worldwide standard is still awaited. Among the recommended Doppler test parameters, the lowest detectable signal could be considered mandatory in Quality Control (QC) protocols for Pulsed Wave (PW) Doppler. Such parameter is defined as the minimum signal level that can be clearly distinguished from noise and therefore, it is considered as related to PW Doppler sensitivity. The present study focuses on proposing and validating a novel image analysis based method for the estimation of the Lowest Detectable Signal in the spectrogram image ( $L D S_{\text {IMG }}$ ), namely Automatic Doppler Sensitivity Measurement Method (ADSMM), as well as to compare its results with the outcomes retrieved from the Naked Eye Doppler Sensitivity Method (NEDSM), based on the mean judgment of three independent observers. Data have been collected from a Doppler flow phantom, through three ultrasound systems for general purpose imaging, equipped with two linear array probes each and with two configuration settings. Results are globally compatible among the proposed methods, US systems and settings. Further studies could be carried out on a higher number of US diagnostic systems, Doppler frequencies and observers, as well as with different probe and phantom models.
\end{abstract}

\section{Section: RESEARCH PAPER}

Keywords: Lowest Detectable Signal; PW Doppler; Automatic Doppler Sensitivity Measurement Method; Quality control; Doppler flow phantom

Citation: Giorgia Fiori, Fabio Fuiano, Andrea Scorza, Jan Galo, Silvia Conforto, Salvatore Andrea Sciuto, A preliminary study on an image analysis based method for lowest detectable signal measurements in Pulsed Wave Doppler ultrasounds, Acta IMEKO, vol. 10, no. 2, article 18, June 2021, identifier: IMEKOACTA-10 (2021)-02-18

Section Editor: Giuseppe Caravello, Università degli Studi di Palermo, Italy

Received January 18, 2021; In final form March 8, 2021; Published June 2021

Copyright: This is an open-access article distributed under the terms of the Creative Commons Attribution 3.0 License, which permits unrestricted use, distribution, and reproduction in any medium, provided the original author and source are credited.

Corresponding author: Giorgia Fiori, e-mail: giorgia.fiori@uniroma3.it

\section{INTRODUCTION}

Pulsed Wave (PW) Doppler Ultrasound (US) is a Doppler technique that allows to display the spectrograms of blood flow velocity from selected depths in tissues. In particular, real-time blood velocity can be accurately measured within a sample volume (SV) in correspondence of a specified depth (adjustable by the operator) from the transducer/medium interface. In this regard, despite the lack of a shared worldwide standard on US equipment testing, performance evaluation of Doppler systems is a currently investigated issue in the scientific research field [1][9]. A great number of Doppler test parameters is recommended by the main medical US professional bodies, to be included in Quality Control (QC) protocols [10]-[12]. Among these parameters, the lowest detectable signal may be considered mandatory to assess PW Doppler system performance, since in scientific literature it is identified as an index of PW Doppler sensitivity [1], [10], [12]-[14]. In particular, the Lowest Detectable Signal in the spectrogram image $\left(L D S_{\text {IMG }}\right)$ has been defined by the authors as the minimum signal level that can be clearly distinguished from noise [15]. In [16], the maximum sensitivity has been referred to as the measurement of the weakest Doppler shift signal (linked to the $L D S_{\text {IMG }}$ through the cosine of the insonification angle) that a US system can detect and display on PW image above the electronic noise. In clinical practice, sensitivity outlines the ability to detect Doppler signals from small vessels for increasing distances from the US probe. The goals of the present study are (a) the improvement of a novel automatic algorithm, namely Automatic Doppler Sensitivity Measurement Method (ADSMM), firstly presented in [15], for the $L D S_{\text {IMG }}$ evaluation by means of a commercial flow phantom; 
(b) its validation through the comparison with the outcomes provided by the Naked Eye Doppler Sensitivity Method (NEDSM) carried out by three observers without clinical expertise. Data have been collected with different settings from three US systems equipped with two linear array US probes each, which worked at similar Doppler frequencies.

In Section 2 the estimation rationale underlying the $L D S_{\text {IMG }}$ parameter definition will be described. In Section 3 the experimental setup used in this study, the ADSMM and the NEDSM implementation will be discussed. In Section 4 the uncertainty analysis of both methods through Monte Carlo Simulation (MCS) will be carried out. In Section 5 results will be presented and discussed on the basis of the comparison between the ones obtained from the ADSMM and the NEDSM, respectively. Finally, in the concluding section the major achievements and the future developments of the research hereby presented will be reported.

\section{LDSIMG ESTIMATION}

In current scientific literature, a shared consensus on the test protocols for the $L D S_{\text {IMG }}$ estimation is still awaited. Nevertheless, factors that affect the lowest detectable signal can be objectively identified. They can be classified in two main groups, according to the device from which they can be adjusted:

a) US system (Doppler frequency $f_{0}$, system settings, sample volume length $S V_{\mathrm{L}}$, sample volume depth $S V_{\mathrm{D}}$, insonification angle $\theta$;

b) test device (Blood Mimicking Fluid BMF, velocity $v$ and reflectors density).

More in detail $f_{0}, v$ and $\theta$ directly affect the Doppler shift $f_{D}$ that determines the PW spectrogram, according to the wellknown (approximated) relationship:

$$
f_{D} \cong 2 f_{0} \frac{v}{c} \cos \theta
$$

Furthermore, in correspondence of the $S V_{\mathrm{L}}$ increase within the flow, the Doppler shift spread increases, as echoes from a higher number of reflectors of different velocities are produced for the same flow. On the other hand, a BMF with higher particles density produces a more intense Doppler signal for the same flow velocity, due to the higher number of reflectors. Moreover, the spectrogram intensity depends on the position of the SV into the tube, depending on the flow velocity profile. Finally, $\mathrm{SV}_{\mathrm{D}}$ determines the spectrogram attenuation: echoes from higher depths are affected by higher attenuation, therefore they are represented in a weaker spectrogram until it can no longer be distinguishable from noise. From the consideration of the abovementioned factors, it is possible to evaluate the $L D S_{\text {IMG }}$ from the sum of two contributions: (a) the attenuation $\Delta \alpha$, due to the echoes path length into the phantom Tissue Mimicking Material (TMM) and (b) the Doppler signal attenuation $\Delta G$ due to the echoes reduction into the spectrogram, from maximum intensity to minimum. Therefore, $L D S_{\text {IMG }}$ can be expressed through the following mathematical formulation:

$$
L D S_{\mathrm{IMG}}=\Delta \alpha+\Delta G \cong 2 \alpha f_{0} S V_{D}+\left(G_{\max }-G_{\min }\right),
$$

where $\alpha$ is the (mean) attenuation coefficient in the TMM (usually expressed in $\mathrm{dB} \cdot \mathrm{cm}^{-1} \cdot \mathrm{MHz}^{-1}$ ), $f_{0}$ is the probe Doppler frequency, $G_{\max }$ is the maximum Doppler gain before no negligible noise appears in the spectrogram image and $G_{\min }$ is the minimum Doppler gain corresponding to the lack of signal (i.e., the spectrogram intensity is very close to zero). Moreover, if the
Table 1. Doppler flow phantom characteristics.

\begin{tabular}{ll}
\hline & Ultrasound Phantom \\
& \\
\hline US phantom model & Gammex Optimizer ${ }^{\circledR} 1425 \mathrm{~A}$ \\
Scanning material & Water-based mimicking gel \\
Tube inner diameter (nominal) & $5 \mathrm{~mm}$ \\
Attenuation & $(0.50 \pm 0.05) \mathrm{dB} \cdot \mathrm{cm}^{-1} \cdot \mathrm{MHz}^{-1}$ \\
TMM speed of sound & $(1540 \pm 10) \mathrm{m} \cdot \mathrm{s}^{-1}$ \\
BMF speed of sound & $(1550 \pm 10) \mathrm{m} \cdot \mathrm{s}^{-1}$ \\
Flow rate (nominal) & $2.6 \mathrm{ml} \cdot \mathrm{s}^{-1}$ \\
Velocity setting (nominal) & $30 \mathrm{~cm} \cdot \mathrm{s}^{-1}$ \\
\hline
\end{tabular}

US system does not provide the Doppler gain in $\mathrm{dB}$ (e.g., arbitrary units, au), a unit conversion is needed.

\section{MATERIALS AND METHODS}

In this work, the image analysis based method for the $L D S_{\text {IMG }}$ estimation, namely Automatic Doppler Sensitivity Measurement Method (ADSMM), implemented in MATLAB environment, has been improved and validated. This has been carried out from the spectrogram images collected from three US systems, equipped with two linear array US probes each, by means of a commercial flow phantom.

\subsection{Experimental setup}

The Gammex, Optimizer ${ }^{\circledR}$ 1425A [17] Doppler flow phantom has been used to acquire the PW Doppler images from a sloped tube within a TMM at a specified continuous flow rate. The device consists of a hydraulic circuit filled with a BMF, a TMM and an electric flow controller (Table 1).

The $L D S_{\text {IMG }}$ measurement has been carried out at two different PW Doppler settings, i.e., set I and set II, and a single Doppler frequency for each linear array probe (Table 2). The lowest and stable flow phantom velocity has been set, and SV size as well as insonification angle have been kept constant. Conversely, the tube inner diameter could not be changed because of the phantom design. Therefore, during the spectrogram acquisitions the $\mathrm{SV}_{\mathrm{D}}$ only has been varied.

Data have been collected for six $\mathrm{SV}_{\mathrm{D}}$ values spaced of $3 \mathrm{~mm}$ each. Figure 1 shows the sample volume depths for each probe.

\begin{tabular}{|c|c|c|}
\hline Parameter & Set I & Set II \\
\hline \multirow{3}{*}{ Dynamic range (dB) } & \multirow{3}{*}{ Maximum } & A: Maximum \\
\hline & & B: 0.8.Maximum \\
\hline & & C: $0.6 \cdot$ Maximum \\
\hline \multirow{3}{*}{ Doppler frequency $(\mathrm{MHz})$} & \multicolumn{2}{|c|}{$A 1: 6.25 ; A 2: 6.25$} \\
\hline & \multicolumn{2}{|c|}{ B1: 5; B2: 6.3} \\
\hline & \multicolumn{2}{|c|}{ C1: 5; C2: 6.2} \\
\hline Wall filter $(\mathrm{Hz})$ & Minimum & $100-150$ \\
\hline \multirow[t]{2}{*}{$\mathrm{SV}_{\mathrm{L}}(\mathrm{mm})$} & \multicolumn{2}{|c|}{ A: $1.5 ; B: 2.0 ; C: 1.5$} \\
\hline & \multicolumn{2}{|c|}{$A 1: 64-79 ; A 2: 52-67$} \\
\hline \multirow[t]{2}{*}{$\mathrm{SV}_{\mathrm{D}}$ range $(\mathrm{mm})$} & \multicolumn{2}{|c|}{ B1: 61-76; B2: 58-73 } \\
\hline & \multicolumn{2}{|c|}{ C1: 73-88; C2: 70-85 } \\
\hline Insonification angle $\left({ }^{\circ}\right)$ & \multicolumn{2}{|r|}{$\sim 51$} \\
\hline
\end{tabular}

Table 2. B-mode and PW Doppler US systems settings.

Set I = raw working conditions; Set II = best working conditions as provided from the specialist. Number 1 , associated to the corresponding US system A, $B$ or $C$, indicates the probe with the lower Doppler frequency, while number 2 indicates the probe with the higher Doppler frequency. 


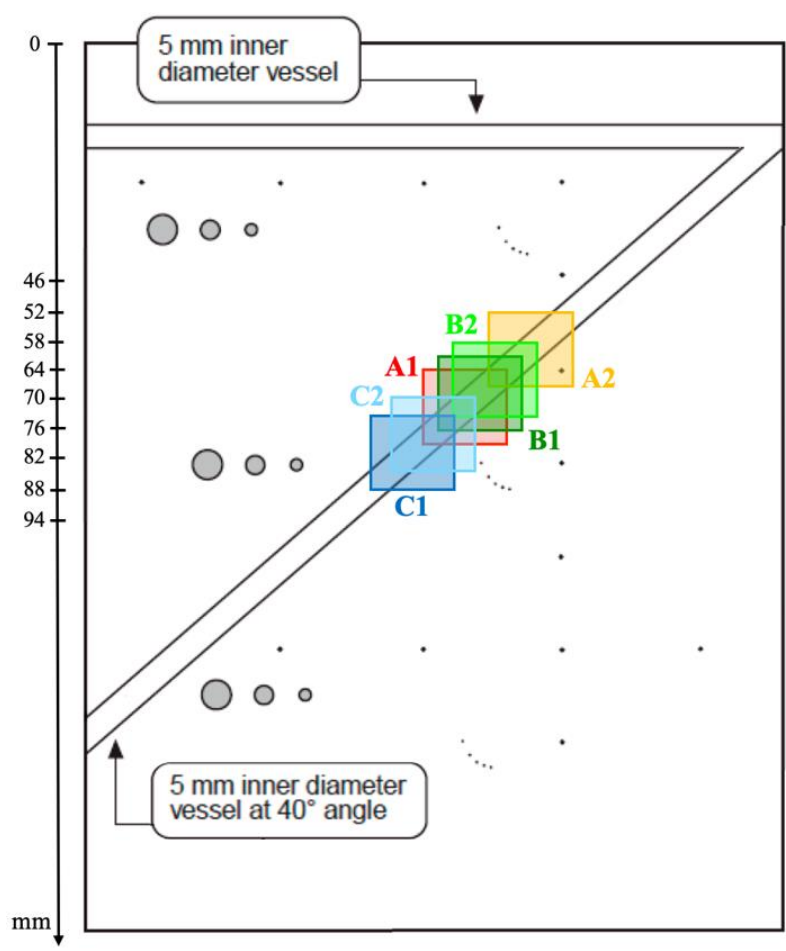

Figure 1. Schematic representation of the sample volume depths according to each US probe. Number 1 , associated to the corresponding US system A, $\mathrm{B}$ or $\mathrm{C}$, indicates the probe with the lower Doppler frequency, while number 2 indicates the probe with the higher Doppler frequency.

Such depths have been set at different values because the nondetectability of the spectrogram intensity varies according to the probe. In the acquisition process, the US probes have been maintained still on the phantom scanning surface through a holder ensuring the insonification angle to be constant throughout the whole acquisition time.

\subsection{Automatic Doppler Sensitivity Measurement Method}

An ad boc acquisition protocol has been designed and implemented to estimate $L D S_{\text {IMG }}$ parameter through the automatic determination of $G_{\min }$ and $G_{\max }$ for each $\mathrm{SV}_{\mathrm{D}}$ value. PW images have been acquired by varying Doppler gain from 0 $\mathrm{dB}$ (or $0 \mathrm{au})$ to the US system maximum adjustable gain with steps of $2 \mathrm{~dB}$ (or $2 \mathrm{au}$ ). After the data acquisition, the ADSMM processes the acquired spectrograms for the automatic determination of $G_{\min }$ and $G_{\max }$ values through two steps:

1) for $G_{\min }$ computation, two Regions of Interest, $\mathrm{ROI}_{\mathrm{s}}$ (signal) and $\mathrm{ROI}_{\mathrm{n}}$ (noise), with the same size $(810 \mathrm{px} \times 144 \mathrm{px})$ are drawn on the PW image in correspondence of the spectrogram and noise, respectively (Figure 2a). The mean gray level value $\mu_{n}$ inside the $\mathrm{ROI}_{\mathrm{n}}$ is calculated to estimate the noise level, while $\mathrm{ROI}_{\mathrm{s}}$ is firstly subdivided in 3240 cells of $6 \mathrm{px} \times 6 \mathrm{px}$ and the mean gray level values $\mu_{s, i}$ of each cell are computed, resulting in a new matrix $\mathrm{ROI}_{\mathrm{s} 2}$. Afterwards, a SNR matrix is obtained whose elements are given by the following expression:

$$
S N R_{i}=\frac{\mu_{s, i}}{\mu_{n}}
$$

SNR matrix is computed for increasing Doppler gain values. Among them, the ADSMM determines $G_{\min }$ as the first gain value for which the number of cells with $S N R_{i} \geq 2$ is higher than $1 \%$ of the total number of cells.

2) $G_{\max }$ is determined from the $\mathrm{ROI}_{\mathrm{n}}$ computed in the first step (Figure $2 \mathrm{~b}$ ). Similarly, it is firstly subdivided into cells of $6 \times 6 \mathrm{px}$
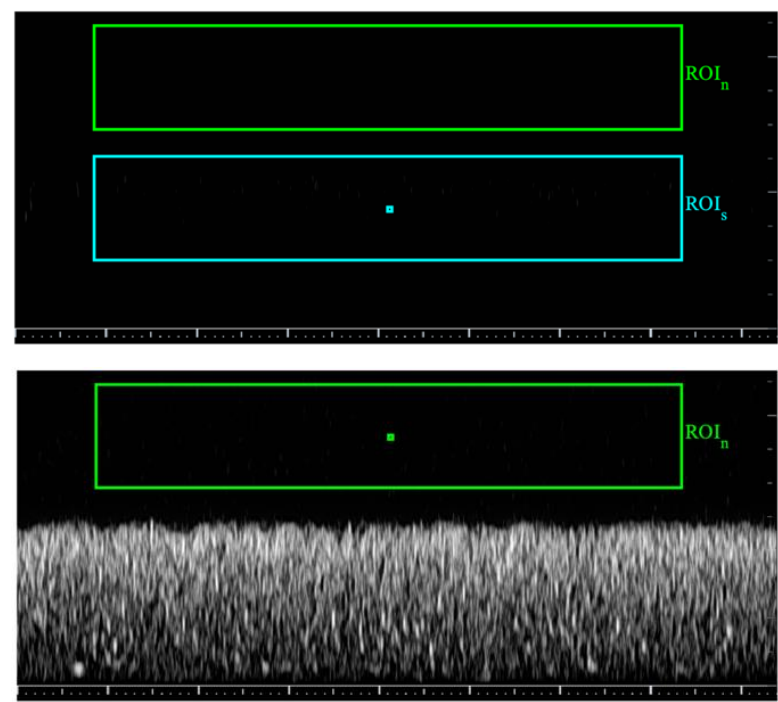

Figure 2. Example of $\mathrm{ROI}_{\mathrm{s}}$ and $\mathrm{ROI}_{\mathrm{n}}$ on $\mathrm{PW}$ spectrograms with $\mathrm{SV}_{\mathrm{D}}$ at $73 \mathrm{~mm}$ in set I configuration, for the automatic determination of a) $G_{\min }$ and b) $G_{\max }$.

and the mean gray level values $\mu_{n, i}$ of each cell are evaluated, resulting in a new matrix $\mathrm{ROI}_{\mathrm{n} 2}$ obtained for increasing Doppler gain values. Among them, the ADSMM determines $G_{\max }$ as the first gain value for which the number of cells with $\mu_{n, i} \geq 3$ is higher than $1 \%$ of the total number of cells.

\subsection{Naked Eye Doppler Sensitivity Method}

The NEDSM is based on an in-house MATLAB function implemented to allow the three observers to express their judgment on $G_{\min }$ and $G_{\max }$ values from the acquired PW spectrograms. Tests have been independently performed without variations of environmental lightening conditions, as well as by keeping a fixed monitor distance. More in detail, the NEDSM randomly provides the observers with the PW spectrograms allowing them to indicate which PW images are associated to $G_{\min }$ and $G_{\max }$ values for each $S V_{\mathrm{D}}$ according to the six linear probes. The PW images order has been randomized and the observers have been requested to repeat the test six times for the study of subjects' inter- and intra-variability.

The compatibility between the results obtained through the ADSMM and the NEDSM has been evaluated according to the following condition [18]:

$$
\left|\mu_{\text {ADSMM }}-\mu_{\text {NEDSM }}\right| \leq \delta_{\text {ADSMM }}+\delta_{\text {NEDSM }},
$$

where $\mu_{\mathrm{ADSMM}}$ and $\mu_{\mathrm{NEDSM}}$ are the mean $L D S_{\mathrm{IMG}}$ values estimated through the ADSMM and the NEDSM, respectively, while $\delta_{\mathrm{ADSMM}}$ and $\delta_{\mathrm{NEDSM}}$ are the corresponding uncertainties.

\section{MONTE CARLO SIMULATION}

As already experienced in other studies [19]-[25], MCS is a powerful tool to estimate the uncertainty and assess the robustness of measurements processed by software. Two different MCS series have been carried out to estimate $L D S_{\text {IMG }}$ uncertainty for the ADSMM and the NEDSM, respectively. The number of iterations for each MCS has been set at $10^{5}$ cycles. The Doppler probe frequency $f_{0}$ uncertainty has been considered negligible because of the narrow bandwidth of the transmitted pulse.

In Table 3 all the distributions assigned to the variables influencing the $L D S_{\text {IMG }}$ expressed in (2), are listed: the TMM 
Table 3. MCS distributions assigned to the variables influencing the $L D S_{\text {IMG }}$

\begin{tabular}{|c|c|c|c|}
\hline Parameter & Distribution & Unit & Mean \pm SD \\
\hline $\begin{array}{l}\text { TMM } \\
\text { attenuation } \alpha\end{array}$ & Normal & $\mathrm{dB} \cdot \mathrm{cm}^{-1} \cdot \mathrm{MHz}^{-1}$ & $0.500 \pm 0.025$ \\
\hline Depth $z$ & Uniform & $\mathrm{cm}$ & $S V_{D} \pm 0.3$ \\
\hline $\begin{array}{l}G_{\min } \text { and } G_{\max } \\
(\mathrm{ADSMM})\end{array}$ & $\begin{array}{l}\text { Uniform } \\
\text { Uniform }\end{array}$ & $\mathrm{dB}$ or $\mathrm{au}\left({ }^{*}\right)$ & $\begin{array}{l}G_{\min , \mathrm{ADSMM}} \pm 0.6 \\
G_{\max , \mathrm{ADSMM}} \pm 0.6\end{array}$ \\
\hline $\begin{array}{l}G_{\min } \text { and } G_{\max } \\
\text { (NEDSM) }\end{array}$ & $\begin{array}{l}\text { Uniform } \\
\text { Uniform }\end{array}$ & $\mathrm{dB}$ or au $\left({ }^{*}\right)$ & $\begin{array}{l}G_{\min , \mathrm{NEDSM}} \pm \sigma_{\min , \mathrm{NEDSM}} \\
G_{\max , \mathrm{NEDSM}} \pm \sigma_{\max , \mathrm{NEDSM}}\end{array}$ \\
\hline
\end{tabular}

$\left({ }^{*}\right)$ The Doppler gain measurement unit is $\mathrm{dB}$ for US systems $\mathrm{A}$ and $\mathrm{B}$, while au for US system $C$. attenuation $\alpha$ has been set as a normal distribution whose standard deviation (SD) has been retrieved from the phantom datasheet by supposing a 95\% confidence level, while the depth $z$ has been set as a uniform distribution with mean value equal to the $\mathrm{SV}_{\mathrm{D}}$ and $\mathrm{SD}$ estimated from the sample volume depth resolution. On the other hand, uniform distributions have been assigned to the ADSMM and the NEDSM minimum and maximum gains. $G_{\min , A D S M m}$ and $G_{\text {max,ADSmm }}$ have been automatically determined by the ADSMM, while $G_{\text {min,NEDSM }}$ and $G_{\max , \text { NEDSM }}$ are the mean values of the three observers judgement. As regards the ADSMM gains standard deviations, they have been estimated considering the Doppler gain resolution. On the

Table 4. $L D S_{I M G}$ results for the ADSMM and the NEDSM according to the US system, probe and configuration setting.

\begin{tabular}{|c|c|c|c|c|c|c|}
\hline \multirow{3}{*}{$\begin{array}{l}\text { US system } \\
\text { and probe }\end{array}$} & \multirow{3}{*}{$\begin{array}{l}\text { Sample volume } \\
\text { depth } S V_{D}(\mathrm{~mm})\end{array}$} & \multicolumn{5}{|c|}{$L D S_{\mathrm{IMG}}(\mathrm{dB})$} \\
\hline & & \multicolumn{3}{|c|}{ Set I } & \multicolumn{2}{|c|}{ Set II } \\
\hline & & & ADSMM & NEDSM & ADSMM & NEDSM \\
\hline \multirow{7}{*}{$\mathrm{A} 1$} & 64 & & $54 \pm 4$ & $55 \pm 4$ & $52 \pm 4$ & $55 \pm 5$ \\
\hline & 67 & & $54 \pm 4$ & $55 \pm 5$ & $54 \pm 4$ & $56 \pm 5$ \\
\hline & 70 & & $54 \pm 5$ & $54 \pm 5$ & $54 \pm 5$ & $55 \pm 5$ \\
\hline & 73 & & $54 \pm 5$ & $53 \pm 5$ & $54 \pm 5$ & $54 \pm 5$ \\
\hline & 76 & & $53 \pm 5$ & $53 \pm 5$ & $54 \pm 5$ & $53 \pm 5$ \\
\hline & 79 & & $55 \pm 5$ & $54 \pm 6$ & $55 \pm 5$ & $53 \pm 5$ \\
\hline & & $L D S_{\mathrm{IMG}, \mathrm{A} 1}$ & $54 \pm 5$ & $54 \pm 5$ & $54 \pm 5$ & $54 \pm 5$ \\
\hline \multirow{7}{*}{$\mathrm{A} 2$} & 52 & & $49 \pm 4$ & $51 \pm 4$ & $51 \pm 3$ & $50 \pm 4$ \\
\hline & 55 & & $48 \pm 4$ & $51 \pm 5$ & $48 \pm 4$ & $51 \pm 4$ \\
\hline & 58 & & $48 \pm 4$ & $50 \pm 4$ & $50 \pm 4$ & $51 \pm 5$ \\
\hline & 61 & & $48 \pm 4$ & $50 \pm 5$ & $46 \pm 4$ & $49 \pm 5$ \\
\hline & 64 & & $46 \pm 4$ & $48 \pm 5$ & $46 \pm 4$ & $47 \pm 5$ \\
\hline & 67 & & $48 \pm 4$ & $50 \pm 5$ & $48 \pm 4$ & $49 \pm 5$ \\
\hline & & $L D S_{\mathrm{IMG}, \mathrm{A} 2}$ & $48 \pm 4$ & $50 \pm 5$ & $48 \pm 4$ & $49 \pm 5$ \\
\hline \multirow{7}{*}{ B1 } & 61 & & $53 \pm 3$ & $52 \pm 4$ & $51 \pm 3$ & $52 \pm 4$ \\
\hline & 64 & & $54 \pm 3$ & $52 \pm 4$ & $56 \pm 3$ & $58 \pm 4$ \\
\hline & 67 & & $56 \pm 4$ & $54 \pm 5$ & $57 \pm 4$ & $59 \pm 4$ \\
\hline & 70 & & $55 \pm 4$ & $55 \pm 5$ & $57 \pm 4$ & $59 \pm 5$ \\
\hline & 73 & & $54 \pm 4$ & $56 \pm 4$ & $55 \pm 4$ & $57 \pm 5$ \\
\hline & 76 & & $54 \pm 4$ & $57 \pm 5$ & $54 \pm 4$ & $56 \pm 4$ \\
\hline & & $L D S_{\mathrm{IMG}, \mathrm{B} 1}$ & $54 \pm 4$ & $54 \pm 5$ & $55 \pm 4$ & $57 \pm 4$ \\
\hline \multirow{7}{*}{ B2 } & 58 & & $59 \pm 4$ & $57 \pm 4$ & $62 \pm 4$ & $64 \pm 5$ \\
\hline & 61 & & $54 \pm 4$ & $56 \pm 5$ & $56 \pm 4$ & $58 \pm 5$ \\
\hline & 64 & & $56 \pm 4$ & $58 \pm 5$ & $56 \pm 4$ & $58 \pm 5$ \\
\hline & 67 & & $58 \pm 4$ & $60 \pm 5$ & $58 \pm 4$ & $60 \pm 5$ \\
\hline & 70 & & $58 \pm 5$ & $60 \pm 5$ & $60 \pm 5$ & $61 \pm 5$ \\
\hline & 73 & & $58 \pm 5$ & $60 \pm 5$ & $60 \pm 5$ & $61 \pm 5$ \\
\hline & & $L D S_{\mathrm{IMG}, \mathrm{B} 2}$ & $57 \pm 4$ & $58 \pm 5$ & $59 \pm 4$ & $60 \pm 5$ \\
\hline \multirow{7}{*}{$\mathrm{C} 1$} & 73 & & $61 \pm 15$ & $67 \pm 19$ & $62 \pm 17$ & $58 \pm 17$ \\
\hline & 76 & & $61 \pm 16$ & $67 \pm 19$ & $62 \pm 17$ & $58 \pm 17$ \\
\hline & 79 & & $61 \pm 15$ & $60 \pm 17$ & $62 \pm 16$ & $63 \pm 18$ \\
\hline & 82 & & $69 \pm 15$ & $70 \pm 18$ & $71 \pm 16$ & $67 \pm 18$ \\
\hline & 85 & & $61 \pm 14$ & $58 \pm 15$ & $56 \pm 9$ & $57 \pm 13$ \\
\hline & 88 & & $61 \pm 13$ & $60 \pm 15$ & $62 \pm 13$ & $61 \pm 15$ \\
\hline & & $L D S_{\mathrm{IMG}, \mathrm{C} 1}$ & $62 \pm 15$ & $64 \pm 17$ & $63 \pm 15$ & $61 \pm 16$ \\
\hline \multirow{7}{*}{$\mathrm{C} 2$} & 70 & & $63 \pm 13$ & $64 \pm 16$ & $57 \pm 8$ & $59 \pm 14$ \\
\hline & 73 & & $63 \pm 13$ & $64 \pm 16$ & $57 \pm 9$ & $59 \pm 14$ \\
\hline & 76 & & $57 \pm 8$ & $57 \pm 10$ & $52 \pm 6$ & $54 \pm 7$ \\
\hline & 79 & & $54 \pm 6$ & $55 \pm 7$ & $56 \pm 7$ & $56 \pm 7$ \\
\hline & 82 & & $54 \pm 6$ & $56 \pm 7$ & $55 \pm 6$ & $56 \pm 7$ \\
\hline & 85 & & $55 \pm 6$ & $55 \pm 6$ & $55 \pm 6$ & $56 \pm 7$ \\
\hline & & $L D S_{\mathrm{IMG}, \mathrm{C2}}$ & $58 \pm 9$ & $59 \pm 11$ & $55 \pm 7$ & $57 \pm 10$ \\
\hline
\end{tabular}


other hand, the standard deviations $\sigma_{\min , \text { NEDSM }}$ and $\sigma_{\text {max,NEDSM }}$ due to subjects' inter- and intra-variability have been obtained through one-way ANOVA statistical test $(p<0.01)$ at each sample volume depth for each US probe.

\section{RESULTS AND DISCUSSION}

The $L D S_{\text {IMG }}$ results for the three US diagnostic systems equipped with two linear array probes obtained through the ADSMM and the NEDSM for set I and set II have been reported in Table 4 and shown in Figure 3. The uncertainty values have been retrieved through 2.5 and 97.5 percentiles from the data distributions obtained through MCSs.

Firstly, by considering the ADSMM, $L D S_{\mathrm{IMG}}$ results are globally compatible for each US system and linear probe, independently from set I and set II (Figure 3 a, c), as well as from $\mathrm{SV}_{\mathrm{D}}$ at which the PW spectrograms have been acquired. More in detail, the results are always compatible in US system A for both its probes throughout the configuration settings, and the same applies for US system C. Conversely, scanner B always shows compatible results for set I only, nevertheless results preserve a global compatibility for set II. The same considerations can be made for the NEDSM, whose $L D S_{\text {IMG }}$ results are globally compatible (Figure $3 \mathrm{~b}, \mathrm{~d}$ ). As regards the comparison between single probe, $L D S_{\text {IMG }}$ values obtained in set I are always compatible for both the methods applied and independently from the sample volume depth. Such compatibility is preserved by changing the configuration setting (set II), except for the first probe of US scanner B, because of the sample volume depth dependency.

Secondly, it's worthy noticing that $L D S_{\text {IMG }}$ outcomes obtained through the ADSMM and the NEDSM, in both set I
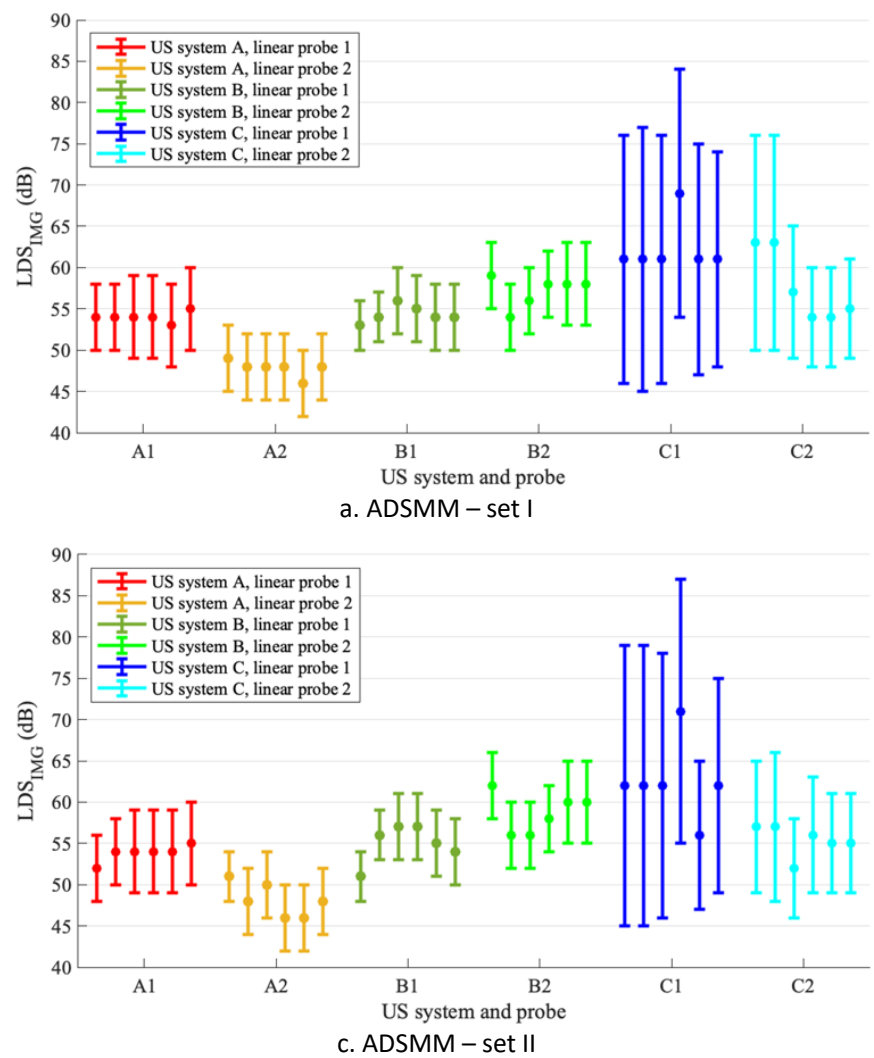
the ADSMM and the NEDSM, it can be noticed that for every

and set II, do not significantly deviate from the constant trend, differently from [15]. Such issue could derive from the reduction of Doppler gain step ( $2 \mathrm{~dB}$ or $2 \mathrm{au}$ ). In fact, the non-constant trend in [15], could have been probably due to the combination of both the further attenuation caused by the presence of nylon pins above the tube sections where PW spectrograms were acquired, and the higher Doppler gain step (5 au) applied, resulting in a wrong identification of $G_{\min }$ and $G_{\max }$.

As regards the methods measurement uncertainty, it can be assessed that the low NEDSM uncertainty values are probably due to an adaptation process that the observer went through during the tests despite the randomization of the PW spectrogram images. Such issue led to a lowering of the intraobserver variability with respect to the inter-observer one. On the other hand, US system $\mathrm{C}$ shows the highest measurement uncertainties, independently from the method applied, because its Doppler gain measurement unit is not provided in $\mathrm{dB}$, leading to the necessity of a specific conversion procedure that introduces a further uncertainty contribution.

Finally, the mean $L D S_{\mathrm{IMG}}\left(L D S_{\mathrm{IMG}, \mathrm{A} 1}, L D S_{\mathrm{IMG}, \mathrm{A} 2}, L D S_{\mathrm{IMG}, \mathrm{B} 1}\right.$, $L D S_{\mathrm{IMG}, \mathrm{B} 2,} L D S_{\mathrm{IMG}, \mathrm{C} 1}$ and $\left.L D S_{\mathrm{IMG}, \mathrm{C} 2}\right)$ and the corresponding uncertainty values for each linear array probe, computed for both the ADSMM and the NEDSM as well as for set I and set II, have been reported in Table 4 and shown in Figure 4.

It should be pointed out that such results always show compatibility among the two methods investigated and the configuration settings. The highest mean $L D S_{\text {IMG }}$ value has been achieved for probe 1 of scanner $\mathrm{C}$ for both methods and configuration settings. Besides, the lowest mean $L D S_{\text {IMG }}$ value has been found in correspondence of the probe 2 of scanner A for both methods and configuration settings. Therefore, the US system $\mathrm{C}$ seems to be the most sensitive among the diagnostic systems involved in the present study.
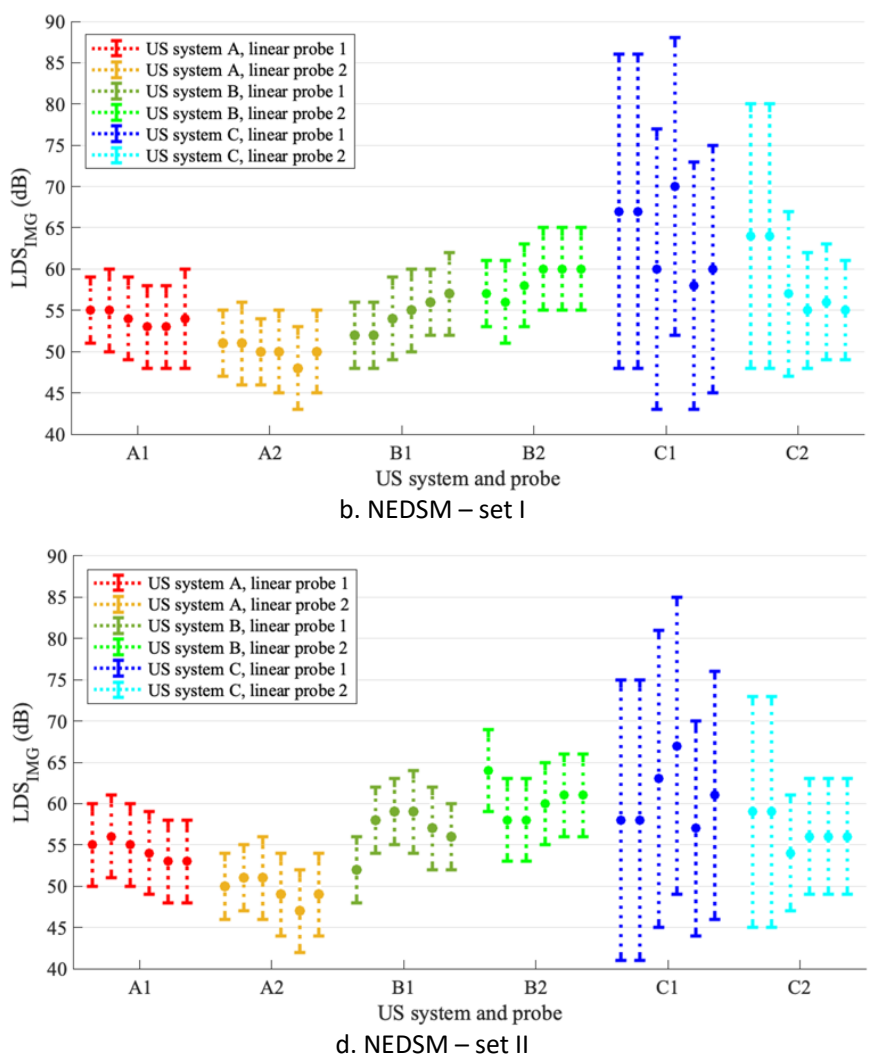

Figure 3. $L D S_{\text {IMG }}$ outcomes obtained through the ADSMM and the NEDSM, for both linear array probes of each US system A, B and C according to the configuration settings (set I and set II). 

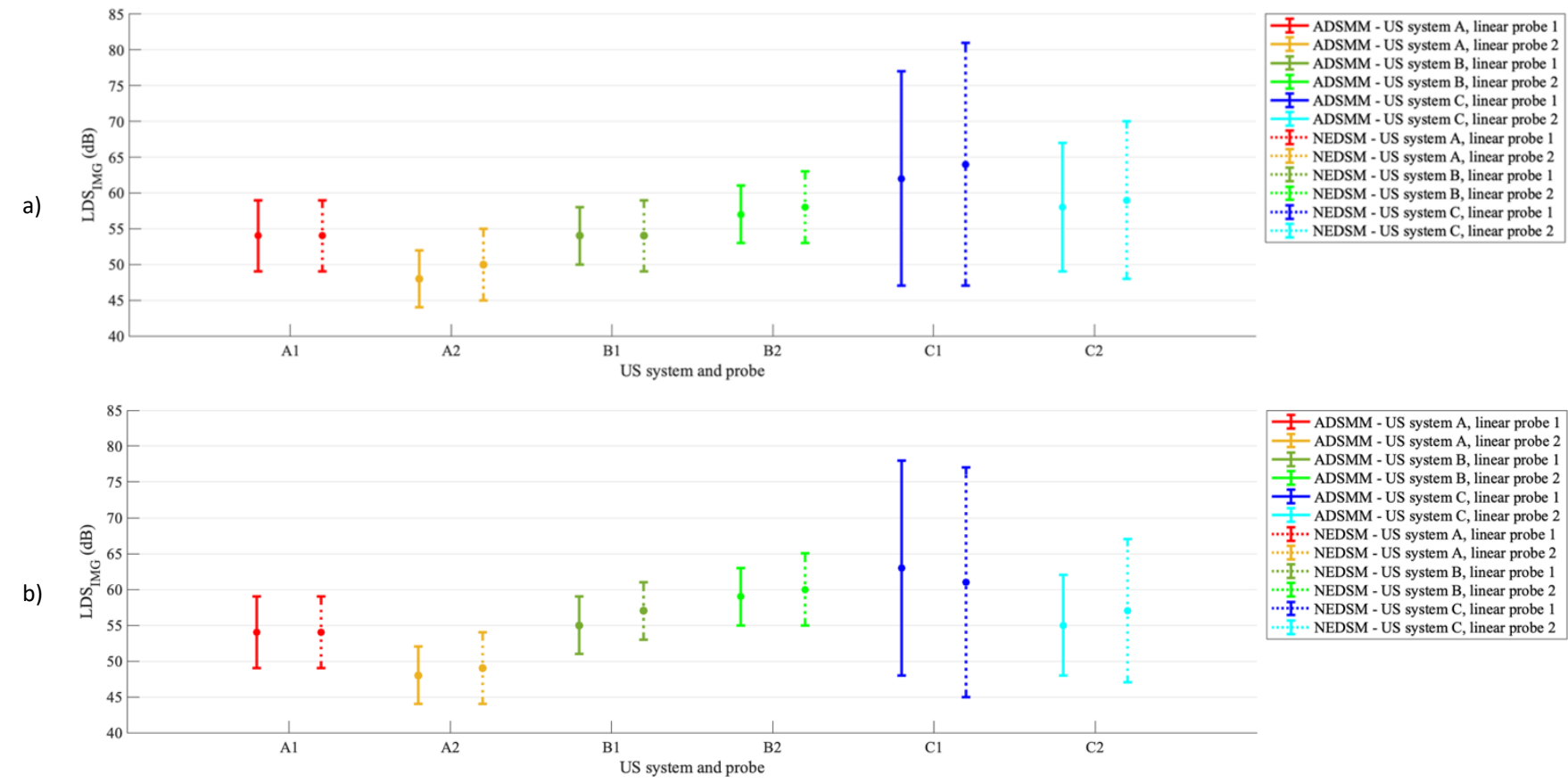

Figure 4. Mean $L D S_{\mathrm{IMG}}$ values ( $L D S_{\mathrm{IMG}, \mathrm{A1}}, L D S_{\mathrm{IMG}, \mathrm{A} 2}, L D S_{\mathrm{IMG}, \mathrm{B} 1,} L D S_{\mathrm{IMG}, \mathrm{B2},} L D S_{\mathrm{IMG}, \mathrm{C1}}$ and $L D S_{\mathrm{IMG}, \mathrm{C2}}$ ) obtained through the $A D S M M$ and the $N E D S M$, for both linear array probes of each US system A, B and C according to a) set I and b) set II.

As a final remark, it should be pointed out that the physical model underlying both the ADSMM and the NEDSM proposed in the present work, is a differential model as it relies on the Doppler gains difference, as in (2). This is undoubtedly an advantage when the observer expresses its own judgment looking at the PW spectrograms: in fact, the model differential nature allows the observer test to be carried out on a monitor with a different dynamic scale with respect to the diagnostic US system one, without affecting the overall results. Such feature improves the NEDSM robustness because tests can be carried out on different monitors.

Further considerations should be addressed about the ADSMM and the NEDSM application in QCs for the assessment of medical US systems, since a significant $L D S_{\text {IMG }}$ reduction with respect to a baseline can be related to both a sensitivity loss $\left(G_{\min }\right.$ tends to increase) and a noise rise $\left(G_{\max }\right.$ tends to decrease). To this aim, the US scanner settings should be adjusted with care, together with the test object speed $v$ in the phantom (e.g., the maximum velocity of the BMF in the flow phantom) and the measurement depth $S V_{\mathrm{D}}$. In particular, both $v$ and $S V_{\mathrm{D}}$ should be selected in order to assure the proper evaluation of $G_{\min }$, since the Doppler signal can be displayed anyway into the spectrogram for higher values of $v$ and low attenuations. In this regard, high sensitivity probes may need deeper test objects embedded in high attenuation TMMs (e.g., steady flows of BMF at higher depths). On the other hand, care should be paid to the test setup and its functioning, in order to avoid any deterioration in its components (e.g., variation in the TMM and/or BMF characteristics) to be confused with performance reduction in the US system. Although the results shown in this work are very promising, all the above issues are worthy of further studies, aiming to provide a suitable and robust tool for QC technicians.

\section{CONCLUSIONS}

In the present work, the Lowest Detectable Signal in the spectrogram image $\left(L D S_{\text {IMG }}\right)$, an index for PW Doppler QC performance test, has been proposed and investigated as it provides an indication about PW Doppler sensitivity. Its estimation has been carried out through an improved image analysis based algorithm, namely Automatic Doppler Sensitivity Measurement Method (ADSMM), developed in MATLAB environment whose physical model has already been proposed in literature. The ADSMM validation has been performed by comparing its outcomes with the ones provided by the Naked Eye Doppler Sensitivity Method (NEDSM), carried out by three independent observers without clinical expertise. Data have been collected from a Doppler flow phantom, by means of three diagnostic systems equipped with two linear probes each, in two different US system settings (set I and set II). Globally, the results obtained through the ADSMM and the NEDSM are compatible, independently from the configuration setting. Such compatibility suggests a good reliability of the ADSMM in $L D S_{\text {IMG }}$ parameter estimation. Among the future developments, further tests could be carried out (a) on a wider observers' sample, (b) on a higher number of US diagnostic systems available in the market, (c) with different probe Doppler frequencies and (d) with different probe models (e.g., convex and phased array). In particular, the latter could be tested on different Doppler phantom models with higher depth size and/or attenuation coefficient to guarantee the correct estimation of the minimum Doppler gain in the $L D S_{\text {IMG }}$ expression. On the other hand, a physical model improvement could be expected in order to retrieve $L D S_{\text {IMG }}$ values even if the Doppler spectrogram is still displayed on the image in correspondence of the zero Doppler gain. Despite the promising results, further studies are going to be carried out in order to assess and improve the robustness and suitability of the $L D S_{\text {IMG }}$ measurement in Ultrasound QC.

\section{ACKNOWLEDGEMENT}

The Authors wish to thank GE Healthcare, HITACHI Healthcare and MINDRAY Medical for hardware supply and technical assistance in data collection. 


\section{REFERENCES}

[1] J. E. Browne, A review of Ultrasound quality assurance protocols and test devices, Phys. Med. 30 (2014), pp. 742-751.

DOI: $10.1016 /$ i.ejmp.2014.08.003

[2] J. M. Thijssen, M. C. van Wijk, M. H. M. Cuypers, Performance testing of medical echo/Doppler equipment, Eur. J. Ultrasound 15 (2002), pp. 151-164. DOI: $10.1016 / \mathrm{s} 0929-8266(02) 00037-\mathrm{x}$

[3] F. Marinozzi, F. P. Branca, F. Bini, A. Scorza, Calibration procedure for performance evaluation of clinical Pulsed Doppler Systems, Measurement 45 (2012), pp. 1334-1342.

DOI: $10.1016 /$ i.measurement.2012.01.052

[4] F. Marinozzi, F. Bini, A. D’Orazio, A. Scorza, Performance Tests of Sonographic Instruments for the Measure of Flow Speed, 2008 IEEE International Workshop on Imaging Systems and Techniques, Crete, Greece, 10 - 12 September 2008. DOI: $10.1109 /$ IST.2008.4659939

[5] A. Scorza, D. Pietrobon, F. Orsini, S. A. Sciuto, A preliminary study on a novel phantom based method for performance evaluation of clinical Colour Doppler systems, Proc. of the 22nd IMEKO TC4 International Symposium \& 20th International Workshop on ADC Modelling and Testing Supporting World Development Through Electrical \& Electronic Measurements, Iasi, Romania, 14 - 15 September 2017. Online [Accessed 14 June 2021].

https://www.imeko.org/publications/tc4-2017/IMEKO-TC42017-033.pdf

[6] A. Scorza, S. Conforto, C. D’Anna, S. A. Sciuto, A Comparative Study on the Influence of Probe Placement on Quality Assurance Measurements in B-mode Ultrasound by Means of Ultrasound Phantoms, Open Biomed. Eng. J. 9 (2015), pp. 164-178. DOI: $10.2174 / 1874120701509010164$

[7] A. Scorza, G. Lupi, S. A. Sciuto, F. Bini, F. Marinozzi, A novel approach to a phantom based method for maximum depth of penetration measurement in diagnostic ultrasound: a preliminary study, Proc. of 2015 IEEE International Symposium on Medical Measurements and Applications (MeMeA), Turin, Italy, 7 - 9 May 2015.

DOI: $10.1109 / \mathrm{MeMeA} .2015 .7145230$

[8] M. Gaitan, J. Geist, B. J. Reschovsky, A. Chijioke, Characterization of laser Doppler vibrometers using acousto-optic modulators, Acta IMEKO 9 (2020) 5, pp. 361-364. DOI: $10.21014 /$ acta imeko.v9i5.1001

[9] G. Dinardo, L. Fabbiano, G. Vacca, How Geometric Misalignments can affect the Accuracy of Measurements by a Novel Configuration of Self-Tracking LDV, Acta IMEKO 3 (2014) 4, pp. 26-31.

DOI: $\underline{10.21014 / \text { acta imeko.v3i4.151 }}$

[10] American Institute of Ultrasound in Medicine, Performance criteria and Measurements for Doppler Ultrasound Devices, AIUM, 2002, ISBN 1-930047-83-5.

[11] Institute of Physical Sciences in Medicine, Report No 70. Testing of Doppler ultrasound equipment, IPSM, 1994.

[12] Z. F. Lu, N. J. Hangiandreou, P. Carson, Clinical Ultrasonography Physics: State of Practice, in: Clinical Imaging Physics: Current and Emerging Practice. E. Samei, D.E. Pfeiffer (editors). Wiley Blackwell, Hoboken, NJ, 2020, ISBN 978-1-118-75345-3, pp. 261286.

[13] D. W. Rickey, A. Fenster, A Doppler ultrasound clutter phantom, Ultrasound Med. Biol. 22 (1996), pp. 747-766. DOI: $10.1016 / 0301-5629(96) 00045-2$

[14] P. R. Hoskins, Simulation and validation of arterial ultrasound imaging and blood flow, Ultrasound Med. Biol. 34 (2008), 693717.

DOI: $10.1016 /$ j.ultrasmedbio.2007.10.017

[15] G. Fiori, F. Fuiano, A. Scorza, J. Galo, S. Conforto, S. A. Sciuto, Lowest Detectable Signal in medical PW Doppler Quality Control by means of a commercial Flow Phantom: a case study, Proc. of the 24th IMEKO TC4 International Symposium \& 22nd International Workshop on ADC Modelling and DAC Modelling and Testing, Palermo, Italy, 14 - 16 September 2020. Online [Accessed 14 June 2021].

https://www.imeko.org/publications/tc4-2020/IMEKO-TC42020-63.pdf

[16] E. J. Boote, J. A. Zagzebski, Performance Tests of Doppler Ultrasound Equipment With a Tissue and Blood-Mimicking Phantom, J. Ultrasound Med. 7 (1988), pp. 137-147.

DOI: $10.7863 /$ jum.1988.7.3.137

[17] Gammex, Optimazer 1425A: Ultrasound Image Analyzer for Doppler and Gray Scale Scanners. Online [Accessed 14 June 2021].

https://cspmedical.com/content/1021086 doppler user manual.pdf

[18] J. R. Taylor, An Introduction to Error Analysis: The Study of Uncertainties in Physical Measurements, University Science Books, Sausalito, CA, USA, 1996, ISBN 978-0-198-55707-4, pp. 13-44.

[19] F. Orsini, S. Scena, C. D’Anna, A. Scorza, L. Schinaia, S. A. Sciuto, Uncertainty Evaluation of a Method for the Functional Reach Test Evaluation by Means of Monte-Carlo Simulation, Proc. of the 22nd IMEKO TC4 International Symposium \& 20th International Workshop on ADC Modelling and Testing Supporting World Development Through Electrical \& Electronic Measurements, Iasi, Romania, 14 - 15 September 2017. Online [Accessed 14 June 2021]. https://www.imeko.org/publications/tc4-2017/IMEKO-TC42017-028.pdf

[20] F. Orsini, F. Fuiano, G. Fiori, A. Scorza, S. A. Sciuto, Temperature influence on viscosity measurements in a rheometer prototype for medical applications: a case study, Proc. of 2019 IEEE International Symposium on Medical Measurements and Applications (MeMeA), Istanbul, Turkey, 26 - 28 June 2019. DOI: $10.1109 / \mathrm{MeMeA} .2019 .8802218$

[21] G. Fiori, F. Fuiano, A. Scorza, M. Schmid, S. Conforto, S. A. Sciuto, ECG Waveforms Reconstruction based on Equivalent Time Sampling, Proc. of 2020 IEEE International Symposium on Medical Measurements and Applications (MeMeA), Bari, Italy, 1 June - 1 July 2020. DOI: $10.1109 / \mathrm{MeMeA} 49120.2020 .9137260$

[22] F. Vurchio, F. Orsini, A. Scorza, F. Fuiano, S. A. Sciuto, A preliminary study on a novel automatic method for angular displacement measurements in microgripper for biomedical applications, Proc. of 2020 IEEE International Symposium on Medical Measurements and Applications (MeMeA), Bari, Italy, 1 June - 1 July 2020 . DOI: $10.1109 / \mathrm{MeMeA} 49120.2020 .9137249$

[23] G. Fiori, F. Fuiano, F. Vurchio, A. Scorza, M. Schmid, S. Conforto, S. A. Sciuto, A preliminary study on a novel method for Depth of Penetration measurement in Ultrasound Quality Assessment, Proc. of the 24th IMEKO TC4 International Symposium \& 22nd International Workshop on ADC Modelling and DAC Modelling and Testing, Palermo, Italy, 14 - 16 September 2020. Online [Accessed 14 June 2021]. https://www.imeko.org/publications/tc4-2020/IMEKO-TC42020-62.pdf

[24] G. Fiori, F. Fuiano, A. Scorza, J. Galo, S. Conforto, S. A. Sciuto, A Preliminary Study on the Adaptive SNR Threshold Method for Depth of Penetration Measurements in Diagnostic Ultrasounds, Appl. Sci. 10 (2020). DOI: $10.3390 /$ app10186533

[25] A. Lavatelli, E. Zappa, Uncertainty in vision based modal analysis: probabilistic studies and experimental validation, ACTA IMEKO 5 (2016), 4, pp. 37-48. DOI: $10.21014 /$ acta imeko.v5i4.426 